nucleus, and some contain smaller cells with nuclei and nucleoli. The shape of these abnormal cells varies as much as does their size; some are round, some oval, some quadrangular or polygonal, some tapering at one end, and some at both ends. With these distorted and fantastic cells, normal epithelium may be mingled; but the normal cells are few in number, while the diseased cells are many. The following sketch represents the scraping from the surface of an epithelial ulcer of the tongue in a man aged 53 , in whom the glands beneath the jaw were extensively diseased. Here, pus-cells, blood-cells, healthy, and diseased epithelial cells, are present.

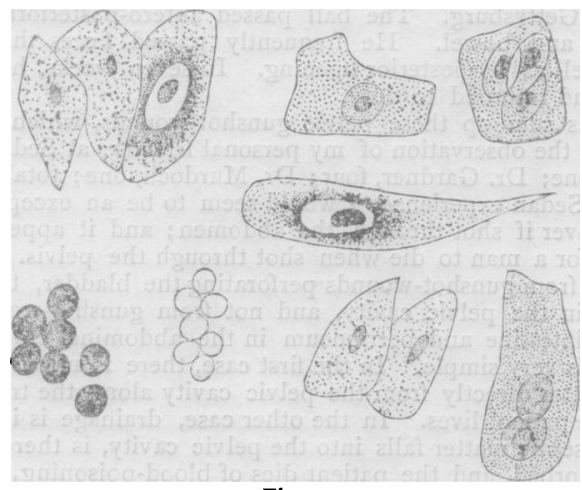

Fig. 1 .

Sometimes, and not unfrequently, even more characteristic structures than any of those described are found; i.e., cell-nests, or portions of cell-nests. Two of these are represented in the accompanying sketch, taken from the scraping of an epithelial ulcer of the tongue in a man forty-three years old.

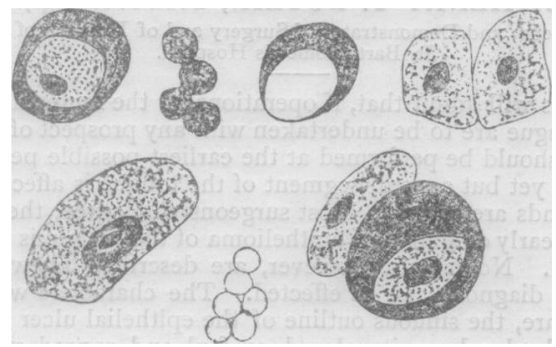

Fig. 2.

I first applied this method of examination in the case of an epithelioma of the tongue in a young man, where the diagnosis was difficult, partly on account of the patient's age (29). The mieroscopic characters of a scraping dispelled all doubt of the nature of the disease. I have since used it in several difficult cases, with the greatest satisfaction. In order to prove that the test is reliable, I have made sections after removal of the epitheliomata which have been scraped and examined before removal, and have thus been sure that the diagnosis was correct. I have also examined scrapings taken from other kinds of ulcers of the tongue, and have never found structures resembling those of epithelioma. Pus-, and blood-, and granulation-corpuscles are present in large quantities. Micrococcus-masses, díbris, and normal epithelium are almost always present; but diseased and altered epithelial cells are not found. The scraping from which this sketch was made was taken from a very destructive syphilitic ulcer of the anterior portion of the tongue of a man. The ulcer healed with exceeding quickness under ten-grain doses of iodide of potassium. (The large granular body is a micrococcus-mass.)

I can, thercfore, confidently state that the structures I have described are found in epithelial carcinoma of the tongue, and that they are not found in any other kind of ulcer with which I am acquainted. I cannot yet say when these structures begin to be formed, and therefore how soon an epithelioma may be recognised by examining a scraping from it. But I know that they are early formed : for I have found them in ulcers of only a few weeks' duration, which few persons would have ventured certainly to diagnose and treat as cancerous, had it not been for their presence.

I do not, of course, allege that this proposition, thus to examine malignant ulcers, is original; but I desire to place it on a firmer footing than hitherto; to show how far it is reliable, and in what cases it should be used.

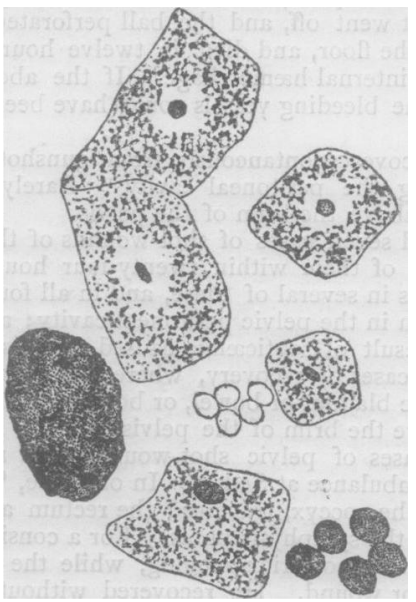

Fig. 3.

The method is very simple, and very easily applied. The surface of the ulcer to be examined is cleaned by lightly brushing it, or gently scraping it with a blunt instrument; a slightly deeper scraping is then taken, mixed with a drop of water on a glass slide, and examined with a quarter-inch power, or No. 7 Hartnack. The pain produced by this operation is so trivial that it seldom calls forth a complaint; and the scraping may be repeated several times, if necessary, without seriously inconveniencing the patient. It may be employed, not only for epithelioma of the tongue, but for squamous epithelioma (squamous-celled carcinoma), whenever it occurs in accessible situations. I have used it for epithelioma of the upper jaw, the face, the penis, and the uterus. I am afraid it cannot be employed, however, for malignant ulcers other than squamous epitheliomas with any degree of certainty; for the structures of which most of them are composed are not sufficiently characteristic to be easily recognised.

One caution may be given. It must not be too hastily assumed that an ulcer is not epitheliomatous because these structures are not at once discovered. If its general characters be suspicious, it should be repeatedly examined before a definite conclusion is arrived at.

I hope it will not be believed, because I recommend so strongly this method of diagnosis, that $I$ am insensible to the general characters of epithelial ulcers, or that I desire others to take no notice of them. On the contrary, I think they should be as carefully studied in the future as in the past; and, when any of them can be shown to be reliable in diagnosis, the microscope should be dispensed with.

\section{AN ANOMALOUS CASE OF INFANTILE HEMI- PLEGIA.}

BY W. B. HADDEN, M.D., M.R.C.P.,

Demonstrator of Morbid Anatomy to St. Thomas's Hospital; Physician to the St. George's and St. James's Dispensary.

THE patient whose history is narrated below was exhibited in the museum at the recent International Medical Congress. The case is one of great interest, both from a pathological and a clinical point of view. The patient is a boy aged 13 , whose family are free from all neurotic tendencies. He has been subject to fits, occurring at intervals of from one to five months or more, since he was one year old. When a child three years of age, he had a fall, since which (according to the friends' statement) he has been paralysed in both legs and right arm.

The fits are preceded by irritability of temper, and by a distinct aura, consisting of a "nasty taste" in the mouth. Movements with trembling then begin in the right hand, and rapidly extend over the rest of the body. During the attacks, he bites his tongue, and foams at the mouth. After the fit, the patient is drowsy, dull, and sometimes so weak that he is confined to his bed for four or five days. With the exception of an attack of chicken-pox, and the presence of worms when he is constipated, the patient has suffered from no other complaint.

The boy was admitted into St. Thomas's Hospital, under Dr. Bristowe, on account of a severe epileptic attack, which left him uncon- 
scious for several hours. When first seen, he was strongly convulsed, and there were clonic spasms of the hands, especially the right, which was rapidly opened and shut. The upper extremities were far more affected than the lower, which, with the exception of a few slight twitches, were motionless. The muscles of the face and eyeballs were strongly convulsed; the pupils constantly varied in size. The patient was quite unconscious. The patellar reflex was said to be entirely absent, although there was marked ankle-clonus. On subsequent examination, the patient was found to be a fairly intelligent boy. Although his friends consicier him somewhat deficient mentally, he reads, counts, and remembers well. His expression is rather vacant, but there is certainly no notable intellectual deficiency. The pupils are equal, and act well to light; the tongue, which is a little tremulous, is protruded straight; there is no sign of facial paralysis. The right upper extremity is much smaller than its fellow, the ulna being an inch shorter than the bone on the other side. The muscles are evidently atrophied to a considerable extent. The fingers of the right hand are kept apart and extended, especially the index and middle fingers. The tendon-reflexes are much more marked than on the left side. There is very pronounced loss of power both of the arm and hand. The left upper extremity is in every respect natural. The legs are rigid, especially the right; and there is well marked talipes equinus on both sides. The patellar reflex is exaggerated, but there is no appreciable difference between the two limbs in this respect. Ankleclonus was usually obtainable on both sides to an equal extent; but occasionally it was impossible to provoke it, in consequence of the great rigidity of the calf-muscles. The lower extremities are not atrophied, and evidently possess considerable muscular force. The plantar, epigastric, abdominal, and cremasteric reflexes are all present; but it is difficult to say whether they are exaggerated or not. Sensation is everywhere intact. There is no atrophy of the thoracic wall or pelvis on either side. The thoracic and abdominal organs are perfectly healthy. But the most striking feature in this patient is his gait. When asked to walk, he stands upright, and supports himself for a moment by leaning against some object. It is evident, indeed, that, although not lacking the muscular force, he is unable to maintain his equilibrium, when upright, without extraneous aid. This is no doubt due to the great contraction of the gastrocnemii and their tendons. Erect, he rests on the ends of the metatarsal bones, the heels being drawn up from the ground; the thighs are adducted, the knees almost in contact ; the legs incline outwards, and the feet are much inverted. When walking, he brings each lower limb forwards by describing an arc with the convexity outwards. At the same time, the head and shoulders are thrown back, the dorsal curve is greatly exaggerated, and the right arm, which is flexed on the forearm, with the corresponding hand dropped on the wrist, is brought up nearly to a level with the chin. As he walks, he keeps the right upper extremity in this position; but the opposite limb is moved backwards and forwards, and, together with the trunk, which sways powerfully from side to side, serves to maintain the equilibrium during progression. The patient's attitude, when standing, is depicted fairly well in the accompanying figure.

In considering this case, the fact that the epileptic attacks begin in the right upper extremity, and affect it more powerfully than the other limbs, strongly suggests that the lesion in this patient is primarily cerebral. But, although this is probably so, the secondary spinal effects are now much more pronounced, and, unfortunately, much more disastrous, than is usually the case in hemiplegia.

What, then, is the probable nature of the cerebral lesion ? and where is it situated? With a fair amount of confidence it may be said that the lesion is for the most part destructive, and that it involves the motor zone of the brain. The circumstance that epileptic attacks still persist suggests that, although the lesion may be to a large extent destructive, certain of the ganglionic cells of the cortex are in a condition of unstable equilibrium, and, by their discharge, give rise to the occasional epileptic attacks.

Furthermore, not only the cortex, but also the subjacent white matter has been involved-if indeed we are to believe the dictates of modern experiment and pathological anatomy. Assuming then that the lesion has involved the motor convolutions (ascending frontal and parietal and paracentral lobule) on the left side, the consequence would be descending degeneration of the pyramidal tract in the hemisphere, crus cerebri, pons and medulla oblongata on that side. In the cord, the direct pyramidal tract (fasciculi of Tiirck) on the same side, and the crossed pyramidal tract in the lateral column of the opposite side, would be degenerated. Clinically, such a change would reveal itself by rigidity, contracture, and exaggeration of the reflexes of the corresponding limbs. It happens-rarely, it is true-that the sound limb in a hemiplegic patient occasionally becomes affected with contracture, like its fellow. The explanation of this curious fact is somewhat difficult. Charcot has put forward the hypothesis that some of the fibres of the pyramidal tract of one side pass through the anterior commissure of the spinal cord to gain the opposite side. In other words, the motor tract decussates, not only at the lower part of the medulla oblongata, but also in the spinal cord itself, to an extent varying probably according to the individual.

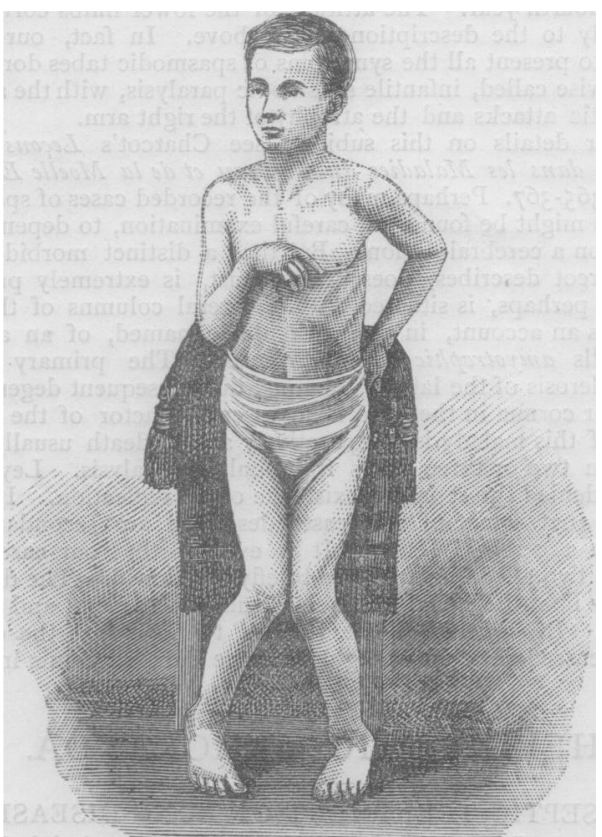

By means of preparations in chloride of gold, Schiefferdecker has shown that this is really so. But further proofs are not wanting. If one half of the spinal cord in a guinea-pig be divided, there is well marked paralysis of the corresponding side and slight paresis of the other side. Again, it is extremely probable that the motor strands are in intimate relation in the cord, when we consider that the lower limbs are constantly associated together in movement. Not unfrequently, when a hemiplegic patient exerts his sound arm by lifting a weight, for example, the paralysed hand, if contractured, at the same time closes spasmodically.

Now, in spite of the belief of our patient's friends that his trouble is dependent on a fall when three years old, we are inclined strongly to the opinion that the primary lesion, as we have before said, is cerebral. It must be remembered that the fits began when he was in his first year, and that he did not walk until his third year-about the time when the accident happened. It may be that the pyramidal tracts, which are in full progress of development from birth up to the first year or later, are more apt to take on degenerative or inflammatory changes at this period of life than subsequently. The wasting of the right upper extremity is doubtless dependent on atrophy of the large multipolar cells of the right anterior cornua at some point of the cervico-brachial enlargement.

Pitres has described an analogous case of muscular atrophy affecting the left arm in a hemiplegic patient. (Arch de Physiologie Normale et Pathologique," 1876, p. 664.) After death, it was found that most of the large cells of the left anterior cornua had disappeared, at the level of the cervico-brachial enlargement, between the seventh and ninth nerve-roots. At present it is impossible to say under what conditions the motor cells become affected with atrophy in cases of lateral sclerosis. Usually, the anterior cornua display great resisting power to the extension of the morbid process from the degenerated pyramidal tracts.

Hemiplegia, when occurring in infancy, frequently entails arrest of development of the paralysed limbs; whereas the same affection when developed in adult life has not the same effect, because the parts are already fully developed. Not unfrequently it is found that the trunk on the paralysed side, in cases of infantile hemiplegia, is imperfectly formed. No such abnormality was observable in our patient.

The case just considered is especially important from a diagnostic point of view. Erb, Seeligmiller; and Stromeyer have called attention 
to a spinal affection, which Charcot has termed spasmodic tabes dorsalis. The disease occurs both in adult and infant life, but in the latter case it presents some peculiarities not seen in the former. The following are the main features of the affection as it occurs in early life : The rigidity begins soon after tirth, and is unaccompanied by cerebral symptoms. The nurse fregitently discovers that the extremities and trunk are rigid, and that the child is dressed with difficulty. Walking is delayed unti the third or fourth year. The attitude of the lower limbs corresponds almost exacil; to the description given above. In fact, our patient may be sajd to present all the symptoms of spasmodic tabes dorsalis, or as it is oth swise called, infantile spasmodic paralysis, with the addition of the epileptic attacks and the atrophy of the right arm.

For further datails on this subject, see Charcot's Lecons sur les Eocalisations dins lis Mraladies du Cervean et de la Moclle Efinive. Part II, ni) 365.367 . Perhaps many of the recorded cases of spasmodic abes dorsalis might be found, on careful examination, to depend, as in our patient, on a cerelral lesion. But that a distinct morbid entity, stich as Charcot describes, does really exist, is extremely probable. The change, perhars, is situated in the lateral columns of the cord. Charcot sives an ascomt, in the work above named, of an affection which he calls amyotrothic lateral sclerosis. The primary change consists of sclerosis of the lateral columns, but subsequent degeneration of the anterior corn:ia in the cord is an essential factor of the disease. The course of this malaty is comparatively acute, death usually superrening within two or three years from bulbar paralysis. Leyden, of Ecrlin, he denied the separate existence of this affection. It is possible indect that some of the cases described are secondary, and devendent on a cerebral lesion. It is evident that our case closely resembles the two affections we have briefly mentioned. The diagnosis from ordinary infantile spinal paralysis is sufficiently clear, and requires no comment. In conclusion, we venture to hope that the case we have just discussed may throw light on some debated points in nervenathology.

\section{THERAPEUTIC MEMORANDA.}

\section{ANTISEPTIC TREATMENT OF LUNG-DISEASE.}

FOR several years, I have largely employed dry antiseptic inhalation in phthisis, as an adjunct to general constitutional measures. The treatment I believe to be useful; but every case of improvement must not be attributed to the inhalation. The most suitable cases are those attended with profuse expectoration, especially when softening has commenced or cavities formed.

The effect is sedative; in a large proportion of cases the expectoration diminishes in quantity and improves in quality, cough becomes less frequent and severe, and sounder sleep is enjoyed, enabling the natient to dispense with objectionable cough medicines. The same affects may be noted when the general progress of the lung-affection is not arrested. I have never seen hamoptysis produced by its use. As a respirator, I prefer a simple tin-box, perforated and shaped to the mouth, introduced by Dr. Roberts of Manchester. The patient is directed to place a few drops of the carbolic solution on the tow in the $\mathrm{b} \cap \mathrm{x}$, and to use the respirator for ten minutes after the morning cough, and at intervals during the day. Many habitually use it for hours while reading. If dryness and irritation of the mouth and throat be caused by 1t:e carbolic inhalation, other remedies may be substituted--such as terebene and eucalyptis oil.

To produce an asentic atmosphere, the constant use of the vapour of arbolic acid in the sicl-room has been recommended; few can be inituced to sulbmit to this treatment, which I cannot recommend. W. V. Sxow, M.D.Lond., Bournemouth.

\section{ON THE TREATMENT OF SOME FORMS OF} PNEUMONIA.

I wisi to draw attention to the remarkable effects produced by the parchloride of iron, combined with hydrosyanic acid, in cases of pneumonia of a low type, especially those due to blood-poisoning. Most practitioners will agree in having seen cases of pneumonia run a course so like, in its general aspect, that of erysipelas as to lead them to imagine that they might be due to a similar cause, taking effect in the interstitial substance of the lung, instead of in the subcutaneous tissue. I have seen many such, and I have begun to apply a similar treatment, with, as I say, truly marvellous effects. The first case of the kind in which I rentured on this treatment was that of Mrs. G. aged 35, who had double pneumonia, with pleurisy on the right side, in Febriary of last year. When I first saw her, the pulse was I 40 , the temperature in the axilla $103^{\circ}$, and the sputa of a deep rust colour. I ordered mustard and linseed poultices, and the following mixture R Liquoris ferri perchloridi fort. $3 \mathrm{ij}$; acidi hydrocyanici (Scheele) $m$ viij;

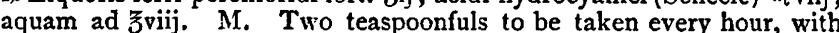
an intervening teaspoonful of brandy in water. After thirty hours, the pulse had fallen to 100 , the temperature to $99^{\circ}$, the sputa were entirely devoid of blood, and the breathing was almost normal. This patient made a rapid recovery.

In the last case of the kind coming under my notice, which occurred last week, the patient seemed to bei n a state of collapse, or syncope; the pulse was 144; the breathing in short gasps; the finger-ends, as seen through the nails, of the colour of a thunder-cloud; and both lungs in a general state of clog. Delirium also lasted a whole night. She had complained of shortness of breath, and had a phthisical aspect and family history, but had never had any cough until the present time. I ventured upon the same treatment with her; and her pulse is now 96 , temperature all but normal, sputa devoid of blood or discoloration of any kind, and she herself anxious to get up.

D. BIDDLE, Kingston-on-Thames.

\section{OBSTETRIC MEMORANDA.}

\section{TRI PLETS.}

THE following case, which has recently occurred in my practice, may be of interest to some of the readers of the Journal. A few day' ago, I was called to attend Mrs. M., aged 34, in her third confinement. The two children by her former confinements are aged four and eight years respectively. At I0.30 A.M., the nurse who came for me stated the "child's feet and body were in the world for some time"; and, on going to the patient, $I$ found it so, and delivered in the usual manner. From the long-continued pressure on the umbilical cord, the child was almost asphyxiated, and I adopted Sylvester's method for resuscitation, together wiih stimulation to the spine, and it gradually revived. On returning to the mother to examine the uterus, I found the head of a second child presenting above the brim of the pelvis, which was also distinctly felt through the parietal wall; but there were no indications of a second labour. I watched her closely all day, waiting the course of events ; and towards night, finding the "pains" were not returning, I adminis. tered a drachm of the liquid extract of ergot; this did not produce the slightest effect, and, there being no immediate symptoms of danger, I decided upon leaving nature to take its course, sustaining her strength with nourishing food and stimulants. At 12 P.M., she complained of aching pain in the back, for which I prescribed a draught containing thirty minims of tincture of opium, after which she had a good night's rest, and was altogether relieved from pain until I P.M. next day, when she again complained of the same pain, which now more resembled the commencement of true labour-pains, and continued so till about 4 P.I., when I thought it advisable to administer another drachm of ergot. Soon afterwards, the pains increased in severity, until the head of the second child presented close to the outlet of the pelvis. She then had violent bearing-down pains for some time; but the head remaining still in the same position, and the patient getting weak, I applied Simpson's forceps, which are the ones I always use, and delivered the child at 6 P.Mr,

On again examining the abdomen, I found that, although it was very much diminished in size, there was still sufficient enlargement to lead me to believe there was another child; and on making a vaginal examination, I found the head of the third presenting. This one was delivered naturally by three or four expulsive pains, and for some time after birth I thought life was extinct; but after adopting the usual methods for artificial respiration, and placing the child in a hot bath, which I think was of the greatest service in this instance, it soon revived.

I kept up the uterine contractions for some time; but, finding they were not sufficien!ly strong to expel the detached placenta, I extracted it in the usual way. There were two distinct placenta, united by a thin membranous band; one of these, somewhat the larger of the two, furnished the supply to two of the cords. The first and third child weighed each six pounds, and the second six pounds and a quarter. J. HowE, L.R.C.S.ED., Castle House, Llanidloes.

BeQUests aNd DoxatroNs. - Mr. Charles Hodgson, of Durham, has bequeathed $£ 3,000$ to the Royal Albert Asylum for Idiots and Imbeciles of the Northern Counties. Mr. Edward Cushee, of the Holloway Road, has bequeathed $\mathcal{E}_{\mathrm{I}, 000}$ consols to the Great Northern Hospital, $£ 500$ consols to the London Hospital, and $£ 500$ consols to the Royal Hospital for Diseases of the Chest. Mr. James Lake of Newlands, Teynliam, has bizueathed $\delta \mathbf{I}, 000$ to the Kent and Canterbury Hospital. 